during the 12 months follow-up. 38 patients (66.67\%) were receiving ETN and 19 (33.3\%) ADA, of which 11 patients $(28.95 \%)$ had a flare with ETN and 8 patients $(42.11 \%)$ with ADA. Table 1 shows demographics. Median time to flare was 5.73 months (IR 2-93-8.9). Concomitant methotrexate was lower in patients with flare (26.32\% vs $71.05 \%)$. In 18 patients (31.58\%), a previous tapering was done and median time of remission before being included was 22 months (IR 15.5-28.5). US does not predict flare in our cohort. Global synovitis score at baseline was 4 (IQR 1.3-10.8) and 0 in BM and PD respectively, and tenosynovitis was 0 both $\mathrm{BM}$ and $\mathrm{PD}$

\begin{tabular}{lcc}
\hline & All patients (n: 57) & Patients with flare (n: 19) \\
\hline Gender; women (\%) & $39(68.42)$ & $10(52.63)$ \\
Time of remission previous to tapering & & \\
$\quad$ median months (IR) & $22(15.5-28.5)$ & $35(35)$ \\
Biologic therapy (ETN/ADA) & $38 / 19$ & $11 / 8$ \\
Concomitant DMARD, $\mathrm{n}(\%)$ & $32(56.14)$ & $5(26.32)$ \\
Previous tapering, $\mathrm{n}(\%)$ & $18(31.58)$ & $5(26.32)$ \\
JIA subcategories: $\mathrm{n}(\%)$ & & \\
Persistent oligoarticular JIA & $15(26.32)$ & $5(33.33)$ \\
Extended oligoarticular JIA & $14(24.56)$ & $4(28.57)$ \\
RF- polyarticular JIA & $15(26.32)$ & $5(33.33)$ \\
RF+ polyarticular JIA & $2(3.51)$ & $1(50)$ \\
Enthesitis related JIA & $3(5.26)$ & $2(66.6)$ \\
Psoriatic JIA & $8(14.04)$ & $2(25)$ \\
\hline
\end{tabular}

Conclusions: Anti-TNF tapering was safe in our JIA patients in more than half of patients after 1 year follow-up. US did not predict flares in our patients. Concomitant treatment with methotrexate was more frequent in patients who remained on remission

References:

[1] Cai Y. Rheumatol Int.2013.

[2] Magni-Manzoni S.Ann Rheum Dis.2013.

Disclosure of Interest: None declared

DOI: 10.1136/annrheumdis-2017-eular.3561

\section{THU0515 DISABILITY AND LOWER QUALITY OF LIFE IS ASSOCIATED WITH SOCIOECONOMIC PASSIVITY IN YOUNG ADULTS WITH JUVENILE IDIOPATHIC ARTHRITIS}

K. Rebane ${ }^{1}$, L. Ristolainen ${ }^{2}$, H. Relas ${ }^{3}$, T. Orenius ${ }^{2}$, H. Kautiainen ${ }^{4}$,

R. Luosujärvi ${ }^{5}, \mathrm{~K}$. Aalto ${ }^{6}, \mathrm{H}$. Säilä ${ }^{2}{ }^{1}$ Children's Hospital, University of Helsinki and Helsinki University Central Hospital; ${ }^{2}$ ORTON Research Institute, ORTON Foundation: ${ }^{3}$ Unit of Primary Health Care and Department of General Practice, Helsinki University Hospital Helsinki; ${ }^{4}$ Helsinki University Central Hospital; ${ }^{5}$ Unit of Primary Health Care and Department of General Practice, Helsinki University Central Hospital; ${ }^{6}$ Children's Hospital, University of Helsinki and Helsinki University Central Hospital, Helsinki, Finland

Background: Health related quality of life of adult patients with juvenile idiopathic arthritis (JIA) is shown to be significantly lower compared to the general population. ${ }^{1,2}$

Objectives: We aimed to recognize young adults with JIA who are socioeconomically passivated and to assess which areas of self-rated health are associated with the emergence of passivity symptoms.

Methods: We studied 195 young adults with JIA using questionnaires addressing demographics, health behavior, and physical activity. The HAQ questionnaire was used to assess functional ability, quality of life was assessed with RAND-36, depressive symptoms were assessed with BDI-II, and self-esteem was evaluated by using the Rosenberg scale. Patients were classified as active if they were engaged in studying, working, maternal leave or military service; and as passive, if they were unemployed or on disability pension.

Results: $80 \%$ of the patients were female, mean age was 23 years, and disease duration was approximately 15 years. Patients in the passive group participated less in leisure time non-physical activities $(p=0.033)$, they felt more disturbed during their leisure time $(p=0.010)$. Leisure time physical activity did not reveal statistically significant differences between the groups. The majority of the patients in the passive group $(58 \%)$ had only basic education $(p<0.001)$, they visited their doctor more frequently $(p=0.019)$ and they used oral prednisolone more often $(p<0.018)$. Approximately $70 \%$ of the patients received disease modifying antirheumatic drugs, and nearly half of the patients were treated with biologicals in both of the groups. Mean disability scores on HAQ were higher in the passive group $(p=0.012)$. Depressive symptoms did not differ between the groups. Selfesteem was lower in the passive group ( $p=0.002)$. Results in health related quality of life revealed statistically significant differences between the groups: physical functioning $(p=0.049)$, social functioning $(p=0.020)$, and emotional well-being $(p=0.047)$ were significantly lower in the passive group.

Conclusions: Patients being socioeconomically more passive showed higher degrees of disability, reporting lower physical functioning, self-esteem, emotional well-being, and social functioning. Those patients should be recognized earlier and activating interventions should be provided.

\section{References:}

[1] Foster HE, Marshall N, Myers A, Dunkley P, Griffiths ID. Outcome in adults with juvenile idiopathic arthritis: A quality of life study. Arthritis Rheum. 2003;48(3):767-75.
[2] Barth S, Haas JP, Schlichtiger J, Molz J, Bisdorff B, Michels H, et al. Long-Term Health-Related Quality of Life in German Patients with Juvenile Idiopathic Arthritis in Comparison to German General Population. PLoS One. 2016;11(4): e0153267.

Disclosure of Interest: None declared

DOI: 10.1136/annrheumdis-2017-eular.5730

\section{THU0516 LONGER TERM OUTCOMES OF CRMO IN A TERTIARY ADOLESCENT AND YOUNG ADULT RHEUMATOLOGY CENTRE IN THE UK}

K.E.N. Clark, F. Josephs, N. Daly, Y. loannou, C.L. Murphy, D. Sen. Rheumatology, University College London Hospitals, London, United Kingdom

Background: Chronic relapsing multifocal osteomyelitis (CRMO) is a rare autoinflammatory bone condition presenting primarily in children and adolescents. It characteristically affects the epiphysis and metaphysis of long bones, and presents with bony pain, local swelling and warmth.

Objectives: The aim of this study was to collate our tertiary adolescent rheumatology centre's experience of managing patients with CRMO, and establish their longer term outcomes, possible by the fact we have a cohort of patients with CRMO under long-term follow-up.

Methods: We carried out a retrospective case note review of all patients who are known to our service with a diagnosis of CRMO, presenting between age 13-20. Results: In total 17 patients were identified as having CRMO, presenting between 1999 and 2015. 10 patients were female, and 7 patients male. The median age of initial symptoms, and age of presentation was 12 years (range1-16 years).

Median duration of follow up is 4.75 years (range $1-16.5$ years). Since initial diagnosis, 3 patients evolved into a SAPHO (synovitis, acne, pustolosis, hyperostosis, osteitis) phenotype, 3 an ERA (enthesitis related arthritis) phenotype and 2 patients developed oligoarticular juvenile idiopathic arthritis (JIA).

$35 \%$ of patients have a purely unifocal disease, and $65 \%$ have multifocal disease as confirmed by whole body MRI. $70.5 \%$ patients had recurrent episodes of inflammation, while $29.5 \%$ of patients had only one flare and then reached remission (either clinical or confirmed with MRI). 15 patients had their diagnosis confirmed with biopsy, while 2 did not due to site of disease. Their diagnosis is assumed based on clinic impression, and typical radiographic findings.

Sites of disease confirmed in our patients include lower limbs (70\% patients), upper limbs (35\% patients), clavicle (29.4\%), mandible (17.6\%) and spine/pelvis (23.5\%).

All patients were treated with NSAIDs. In terms of treatments used since diagnosis, $76 \%$ patients have been on methotrexate (MTX), $47 \%$ had one infusion of pamidronate, and $23 \%$ required more than one infusion of pamidronate. Other medications include sulfasalazine (SSZ), azathioprine, risedronate and anti-TNFs (adalimumab, etanercept and infliximab).

On last clinic review, with or without imaging, $35 \%$ of patients continue to have active disease. Currently $29 \%$ patients are on MTX alone, $23 \%$ patients are on adalimumab and MTX, and $35 \%$ are only maintained on NSAIDS. Of those without active disease 5 patients $(45 \%)$ are not on any DMARD or biologic therapy.

Conclusions: We present here our experience of managing adolescent patients with CRMO.

The perceived wisdom is that CRMO is a self-limiting disease which eventually goes into remission. However our centre's experience is that nearly $50 \%$ of our patients have a disease which evolves into another systemic autoimmune disease, mainly SAPHO, polyarticlar or enthesitis related JIA. Previous case series have suggested only $0-30 \%$ of patients' disease evolves. This may be a reflection of our older cohort of patients, who are only referred to our service with ongoing disease.

The majority of patients have a recurrent and multifocal course of disease. The most common site of disease was in the lower limbs. All patients were treated with NSAIDS, and a combination of DMARDS, bisphosphonates and biologic agents have been used, which has resulted in remission of disease in the majority of patients.

Disclosure of Interest: None declared

DOI: 10.1136/annrheumdis-2017-eular.5188

\section{THU0517 LARGE VESSEL VASCULITIS IN INFANTS - A CASE SERIES}

L. Das $^{1}$, J.H.T. Tan ${ }^{1}$, X.C. Gao ${ }^{1}$, S.F. Hoh ${ }^{1}$, T. Arkachaisri ${ }^{1,2} .{ }^{1}$ Rheumatology and Immunology Services, Department of Paediatric Subspecialties, KK Women's and Children's Hospital; ${ }^{2}$ Duke-NUS Medical School, Singapore, Singapore

Background: Large vessel involvement following recrudescent or recalcitrant Kawasaki Disease or other vasculitidies in young children have been limited to few case reports and outcomes are still unclear.

Objectives: To describe and compare the characteristics and outcomes of 6 patients with large vessel vasculitis diagnosed between 2013 to 2015 in KK Hospital, Singapore

\section{Methods:}

Demographic and disease characteristic information were collected and median, interquartile range (IQR) \& percentiles were used to describe the data.

Results: 6 patients were included in the analysis. Median age was 3.75 months 